\title{
Radiomics Assessment of SPAIR and STIR MRI Sequences to Predict Axial and Peripheral Spondyloarthritis
}

\author{
Ariane P. M. Tenório, José R. Ferreira Jr., Vitor F. Dalto, Matheus C. Faleiros, \\ Rodrigo L. Assad, Marcello H. Nogueira-Barbosa, Paulo M. Azevedo-Marques \\ Faculdade de Medicina de Ribeirão Preto - Universidade de São Paulo \\ Av. Bandeirantes 3900, 14048-900, Ribeirão Preto - SP - Brazil \\ \{tenorioapm, jose.raniery\}ealumni.usp.br \\ \{fdalto, matheuscalil2, rodluppinoassad\}egmail.com \\ \{marcello, pmarques\}efmrp.usp.br
}

\begin{abstract}
In an attempt to aid the subtyping of spondyloarthritis (SpA), this work assessed neural nets and magnetic resonance imaging (MRI) features to predict SpA. Patients underwent SPAIR and STIR MRI sequences. Radiologists manually segmented sacroiliac joints images for extracting MRI features. A neural net used these features to predict SpA. The STIR-based model yielded higher performance than SPAIR to diagnose SpA, although no statistical difference was found between them. The SPAIR model yielded an area under the curve of 0.83 to differentiate axial and peripheral subtypes, while STIR yielded 0.57 ( $p<0.05$ on curves difference). Therefore, neural nets modeled with SPAIR-extracted features distinguished SpA using a single MRI exam of the sacroiliac joints.
\end{abstract}

\section{Introduction}

Spondyloarthritis (SpA) comprises a set of diseases sharing common clinical manifestations, such as inflammatory axial pain, enthesopathy, and peripheral arthritis, which can cause joint damage to work disability [Lambert et al. 2016]. Treatment protocol for SpA considers, among other factors, the subtype of it as axial or peripheral, which makes the diagnosis and subclassification crucial for the therapy decision making [Malaviya et al. 2017].

Magnetic resonance imaging (MRI) plays an important role in the diagnosis and follow-up of SpA. This technique can identify key findings for SpA, like subchondral bone marrow edema, which corresponds to an early stage of the disease [Sieper et al. 2009]. The sequences short tau inversion recovery (STIR) and spectral attenuated inversion recovery (SPAIR) are the current MRI standards for SpA evaluation (Figure 1) [van der Heijde et al. 2017].

In this era of personalized medicine, radiomics has markedly grown as a precision imaging tool for the improvement of diagnosis, assessment of prognosis, and prediction of therapy response [Gillies et al. 2016]. Radiomics currently consists of modeling quantitative features from medical images into a machine-learning method to improve clinical decision support systems [Ferreira Junior et al. 2020]. Once the model has been designed, it can be used as a radiomics-aided support system for precision medicine and SpA evaluation. 

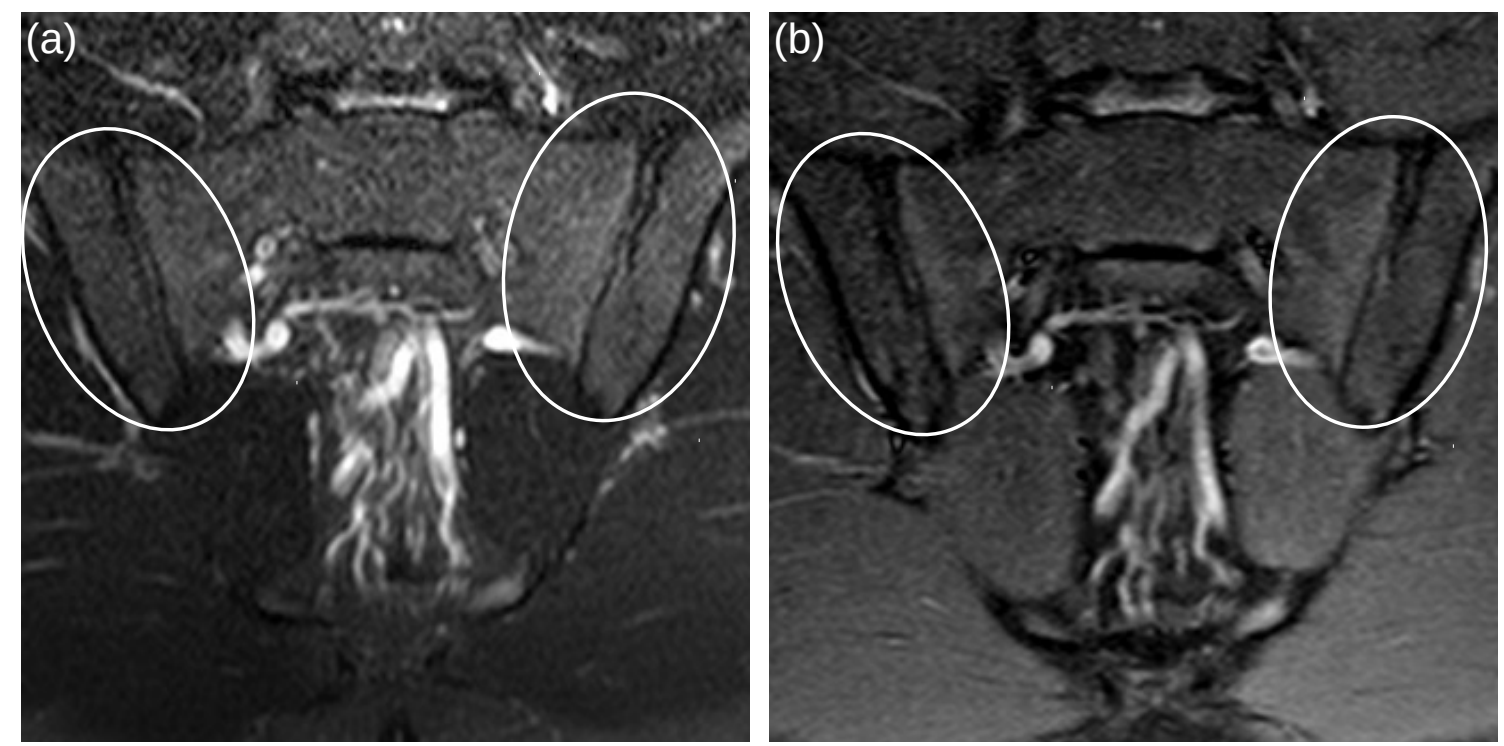

Figure 1. Example of images from the same patient on different MRI sequences for SpA assessment: (a) SPAIR, (b) STIR. Sacroiliac joints are in circles.

Therefore, this work performed a radiomic study to develop machine-learning models using quantitative MRI features from sacroiliac joints to aid the early diagnosis and subtyping of SpA. Besides that, this work also compared statistically the performance of neural networks modeled with features extracted from both MRI sequences of SPAIR and STIR on the prediction of SpA. Previous works have already recognized inflammatory patterns from sacroiliac joints to predict $\mathrm{SpA}$, but they used a single MRI sequence (either STIR or SPAIR), and hence, did not compare the performances of different imaging acquisition protocols [Faleiros et al. 2020, Tenorio et al. 2020].

\section{Material and Methods}

We performed a retrospective study approved by our institutional review board ${ }^{1}$ with a waiver of the patient's informed consent. This study comprised 47 patients with suspected SpA who underwent MRI examinations. All 47 were imaged with SPAIR and 45 patients with STIR. A diagnosis of SpA was established after clinical and laboratory follow-up using additional radiography when necessary [Sieper et al. 2009].

After anonymization, this work selected six consecutive images from each MRI exam to be used in the analysis, as previously used in other studies [Faleiros et al. 2020, Tenorio et al. 2020]. Each image had the sacroiliac joints manually segmented by a musculoskeletal radiologist and processed by the warp geometric transform for feature extraction. A total of 115 first-, second-, and higher-order texture features were extracted from each segmented image, and each exam was characterized by the mean and standard deviation of each feature value obtained, resulting in 230 features per patient. Table 1 lists all variables calculated for this radiomic analysis.

A machine-learning algorithm based on the ReliefF feature selection method and radial basis function network (RBFN) used the quantitative MRI features as input to pre-

\footnotetext{
${ }^{1}$ Comitê de Ética em Pesquisa, Hospital das Clínicas da Faculdade de Medicina de Ribeirão Preto, Universidade de São Paulo, Parecer n ${ }^{0}$ 2.356.447.
} 
Table 1. List of quantitative texture features extracted from each MRI image. The mean and standard deviation were calculated for each variable, resulting in 230 extracted features per patient.

\begin{tabular}{lll}
\hline Type & Category & Features \\
First-order & Histogram & $\begin{array}{l}\text { Mean, Variance, Standard deviation, Coefficient of devia- } \\
\text { tion, Kurtosis, Skewness, Maximum value. }\end{array}$ \\
\hline Second-order & Haralick & $\begin{array}{l}\text { Angular second moment, Contrast, Correlation, Variance, } \\
\text { Inverse difference moment, Sum average, Sum variance, } \\
\text { Sum entropy, Entropy, Difference of variance, Difference } \\
\text { of entropy, Information correlation (2 measures), Maxi- } \\
\text { mum correlation coefficient. }\end{array}$ \\
& Tamura & Contrast, Granularity, Directionality (16 orientations). \\
\hline Higher-order & Gaurier & $\begin{array}{l}\text { Mean, Variance, Standard deviation, Coefficient of devia- } \\
\text { tion, Kurtosis, Skewness, Maximum value. }\end{array}$ \\
& Wavelet & Energy, Standard deviation. \\
& Fractal & Dimension estimation. \\
\hline
\end{tabular}

dict the diseases. This algorithm finds the highest performance for the combination of $x$ selected features and $y$ RBFN hidden-layer neurons, which $x$ varied from 1 to all 230 features, and $y$ varied from 1 to the number of samples of the majority group investigated, as already previously employed [Tenorio et al. 2020].

The area under the receiver operating characteristic (ROC) curve (AUC) assessed the analysis using the leave-one-out cross-validation method. The DeLong's test measured the statistical difference in ROC curves. A test with $p<0.05$ was considered statistically significant. Statistical analysis was performed with the R v3.4.4 package.

\section{Results}

The SPAIR-based model selected 32 quantitative MRI features to distinguish SpA and other diseases (i.e., fibromyalgia, osteoarthritis, or mechanical bone injury), from which 30 were extracted after the application of Gabor filters on the image. This SPAIR model yielded an AUC of 0.623 (95\% confidence interval - CI: 0.469-0.777).

On the other hand, using two STIR-extracted features, i.e., variance and standard deviation calculated after the application of a fast Fourier transform on the image, the STIR model yielded higher AUC of 0.764 (CI: $0.591-0.937$ ), but no statistically significant difference was identified.

To distinguish axial and peripheral subtypes, the machine-learning model yielded AUCs of 0.831 (CI: 0.677-0.986) using the SPAIR sequence (with 26 selected features) and 0.573 (CI: $0.413-0.734)$ with 173 selected STIR-extracted features. A statistically significant difference between their ROC curves was found $(p<0.05)$. 
All mutual features selected by both SPAIR and STIR MRI sequence models developed to predict the subtype of SpA were Wavelet_HL3_M, Histogram_Skewness_M, Tamura_D4_SD, Tamura_D9_SD, Tamura_D9_M, Tamura_D1_M, Tamura_D8_M, Tamura_D11_M, Tamura_D10_M, Tamura_D2_M, Tamura_D7_M, Tamura_D5_M, and Tamura_D6_M. The texture directionalities from Tamura were markedly selected by the ReliefF method. No mutual features were used by the MRI sequences models to distinguish SpA from other diseases.

\section{Discussion}

The diagnosis of SpA in its early stage and the rapid referral of the individual affected by SpA are essential for a better therapeutic result and patient prognosis [Sieper et al. 2009]. There is great difficulty in diagnosing SpA because of its various forms of presentation, requiring several clinical and laboratory assessments that may not be able to be visually performed on the MRI [Lambert et al. 2016]. Radiomics, by contrast, can capture additional information from the MRI that can predict clinical outcomes [Gillies et al. 2016, Santos et al. 2019]. Radiomics could be a tool to aid physicians in clinical decisions because it can identify fine texture details on medical images. Based on the analysis here presented, it is feasible to use radiomics models applied to the MRI assessment of sacroiliac joints to support the diagnosis and subtyping of SpA.

In general, several first-order, second-order, and higher-order texture features were significantly identified for the clinical outcomes studied, showing the potential of texture MRI features to identify SpA patterns. Moreover, the developed radiomic models were able to distinguish the subtypes of the disease using a single imaging exam of the pelvic girdle region. SPAIR and STIR fat suppression sequences were essential to machine learning and SpA association, corroborating with the current clinical protocol, which uses both sequences for SpA evaluation [Lambert et al. 2016, van der Heijde et al. 2017].

The study has limitations that deserve mention. First, this was a retrospective study with a small dataset. Due to its retrospective nature, some clinical parameters could not be obtained, which could improve machine learning. Moreover, external validation datasets were not included. However, this study performed cross-validation to decrease the limitation of the lack of an independent external validation dataset.

Furthermore, this work still needed a prior image segmentation step in order to perform machine learning. Here, the sacroiliac joint segmentation was performed manually, which was a costly process for the radiologists. Therefore, an automatic method for image representation and training of neural networks could bring significant benefits to the radiomics approach. One alternative to it is deep learning and convolutional neural networks. Deep learning features are extracted directly from MRI pixels/voxels without relying on image segmentation and handcrafted feature extraction methods [Santos et al. 2019, LeCun et al. 2015]. Deep convolutional neural networks have been shown an efficient method in medical image analysis and radiomics [Litjens et al. 2017, Lee et al. 2017], and hence, they are intended to be used in future works and compared with the developed RBFN models. 


\section{Conclusion}

The proposed radiomics approach was able to distinguish axial and peripheral subforms of SpA. Radiomics could identify SpA even when active inflammation on sacroiliac joints was not present in the MRI. The results encourage future studies to investigate radiomics texture-based MRI parameters as potential biomarkers. For future directions, we propose to extend the analysis for patient prognostic assessment and therapy response prediction, including the evaluation with deep learning.

\section{Acknowledgements}

This study was financed in part by the Coordenação de Aperfeiçoamento de Pessoal de Nível Superior (CAPES) - Finance Code 001 and by The São Paulo Research Foundation (FAPESP) - grants \# 16/17078-0 and 14/50889-7. PM Azevedo-Marques is also supported by Conselho Nacional de Desenvolvimento Científico e Tecnológico (CNPq) grant \# 305124/2018-8.

\section{References}

Faleiros, M. C., Nogueira-Barbosa, M. H., Dalto, V. F., Ferreira-Junior, J. R., Tenorio, A. P. M., Assad, R. L., Louzada-Junior, P., Rangayyan, R. M., and Azevedo-Marques, P. M. (2020). Machine learning techniques for computer-aided classification of active inflammatory sacroiliitis in magnetic resonance imaging. Advances in Rheumatology, $60: 25$.

Ferreira Junior, J. R., Santos, M. K., Tenório, A. P. M., Faleiros, M. C., Cipriano, F. E. G., Fabro, A. T., Näppi, J., Yoshida, H., and Azevedo Marques, P. M. (2020). CTbased radiomics for prediction of histologic subtype and metastatic disease in primary malignant lung neoplasms. International Journal of Computer Assisted Radiology and Surgery, 15:163-172.

Gillies, R., Kinahan, P., and Hricak, H. (2016). Radiomics: Images are more than pictures, they are data. Radiology, 278(2):563-77.

Lambert, R. G., Bakker, P. A., van der Heijde, D., Weber, U., Rudwaleit, M., Hermann, K.-G. A., Sieper, J., Baraliakos, X., Bennett, A., Braun, J., et al. (2016). Defining active sacroiliitis on MRI for classification of axial spondyloarthritis: update by the ASAS MRI working group. Annals of the Rheumatic Diseases, 75(11):1958-1963.

LeCun, Y., Bengio, Y., and Hinton, G. (2015). Deep learning. Nature, 521(7553):436444.

Lee, J., Jun, S., Cho, Y.-W., Lee, H., Kim, G. B., Seo, J. B., and Kim, N. (2017). Deep learning in medical imaging: general overview. Korean Journal of Radiology, 18(4):570-584.

Litjens, G., Kooi, T., Bejnordi, B. E., Setio, A. A. A., Ciompi, F., Ghafoorian, M., van der Laak, J. A., van Ginneken, B., and Sánchez, C. I. (2017). A survey on deep learning in medical image analysis. Medical Image Analysis, 42:60-88.

Malaviya, A. N., Rawat, R., Agrawal, N., and Patil, N. S. (2017). The nonradiographic axial spondyloarthritis, the radiographic axial spondyloarthritis, and ankylosing spondylitis: the tangled skein of rheumatology. International Journal of Rheumatology, 2017. 
Santos, M. K., Ferreira Junior, J. R., Wada, D. T., Tenório, A. P. M., Barbosa, M. H. N., and Azevedo Marques, P. M. (2019). Artificial intelligence, machine learning, computer-aided diagnosis, and radiomics: advances in imaging towards to precision medicine. Radiologia Brasileira, 52(6):387-396.

Sieper, J., Rudwaleit, M., Baraliakos, X., Brandt, J., Braun, J., Burgos-Vargas, R., Dougados, M., Hermann, K., Landewe, R., Maksymowych, W., et al. (2009). The assessment of spondyloarthritis international society (asas) handbook: a guide to assess spondyloarthritis. Annals of the Rheumatic Diseases, 68(Suppl 2):ii1-ii44.

Tenorio, A. P. M., Faleiros, M. C., Ferreira Junior, J. R., Dalto, V. F., Assad, R. L., Louzada-Junior, P., Yoshida, H., Nogueira-Barbosa, M. H., and Azevedo-Marques, P. M. (2020). A study of MRI-based radiomics biomarkers for sacroiliitis and spondyloarthritis. International Journal of Computer Assisted Radiology and Surgery, DOI:10.1007/s11548-020-02219-7.

van der Heijde, D., Ramiro, S., Landewé, R., Baraliakos, X., Van den Bosch, F., Sepriano, A., Regel, A., Ciurea, A., Dagfinrud, H., Dougados, M., et al. (2017). 2016 update of the ASAS-EULAR management recommendations for axial spondyloarthritis. Annals of the Rheumatic Diseases, 76(6):978-991. 